\title{
Efficacy of a multifactorial strategy for bowel preparation in diabetic patients undergoing colonoscopy: a randomized trial
}

Authors

Institutions
Marco Antonio Alvarez-Gonzalez ${ }^{1,2}$, Juana A. Flores-Le Roux ${ }^{2,3}$, Agustin Seoane ${ }^{1}$, Juan Pedro-Botet ${ }^{2,3}$, Laura Carot ${ }^{1}$, Agnés Fernandez-Clotet ${ }^{1}$, Agnés Raga ${ }^{1}$, Miguel A. Pantaleon ${ }^{1,2}$, Luis Barranco ${ }^{1}$, Felipe Bory ${ }^{1}$, Vicente Lorenzo-Zuñiga ${ }^{2,4}$ submitted 21. January 2016 accepted after revision 6. June 2016

\section{Bibliography}

Dol http://dx.doi.org/ 10.1055/s-0042-111320 Published online: 4.8.2016 Endoscopy 2016; 48: 10031009

(c) Georg Thieme Verlag KG Stuttgart · New York ISSN 0013-726X

\section{Corresponding author} Marco A. Alvarez-Gonzalez, MD

Unidad de Endoscopia Digestiva Hospital del Mar 08003 Barcelona

Spain

Fax: +34-93-2483376

marcoalgo@gmail.com

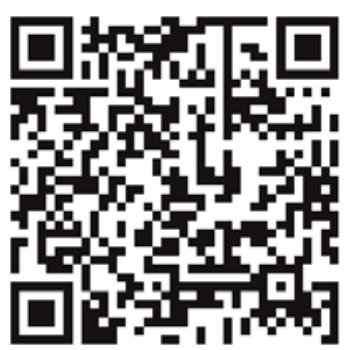

Scan this QR-Code to watch the video comment.
Passeig Maritim 25-29

Background and study aims: Previous studies have reported that diabetes mellitus is an independent risk factor for inadequate bowel preparation. Current guidelines do not recommend a specific preparation for this patient population. The aims of this study were to assess the efficacy, safety, and tolerability of an adapted preparation protocol for colon cleansing in patients with type 2 diabetes mellitus.

Patients and methods: This randomized, singleblind, parallel group, superiority trial compared a conventional bowel preparation protocol (CBP) with a diabetes-specific preparation protocol (DSP). The CBP included a low-fiber diet for 3 days followed by a clear liquid diet for 24 hours before colonoscopy. The DSP included a multifactorial strategy combining an educational intervention, a low-fiber diet, and adjustment of blood glucose-lowering agents. All patients received $4 \mathrm{~L}$ of a polyethylene glycol solution in a split-dose regimen. The endoscopists were blinded to the preparation protocol. The primary outcome measure was inadequate bowel preparation ac-

\section{Introduction}

$\nabla$

The need for colonoscopy is common among patients with type 2 diabetes mellitus owing to the high prevalence of gastrointestinal symptoms and increased risk of colon cancer in this population [1]. Previous studies have reported that diabetes mellitus is an independent risk factor for inadequate bowel preparation [2-4]. The diagnostic yield of colonoscopy depends on the quality of colonic cleansing [5]. In this respect, poor bowel preparation leads to suboptimal colonoscopy, resulting in overlooked disease and unnecessary repeat procedures.

Bowel preparation for colonoscopy requires diet modifications followed by a laxative. Dietary re- commendations usually include a low-fiber diet for 1-4 days, followed by a clear liquid diet for cording to the Boston Bowel Preparation Scale. Secondary outcome measures included hypoglycemic events, tolerability, and acceptability. Results: A total of 150 patients were included in the study (74 CBP and 76 DSP). Both groups were comparable in terms of baseline characteristics. Inadequate bowel cleansing was more frequent following CBP than DSP (20\% vs. $7 \%, P=0.014$; risk ratio $3.1,95 \%$ confidence interval $1.2-8$ ). Only CBP and performance status were independently associated with inadequate bowel preparation. Both preparations were equally tolerated and accepted by patients, and side-effects were similar between the groups.

Conclusions: A multifactorial strategy for bowel preparation in patients with diabetes undergoing colonoscopy showed a threefold reduction in the rate of inadequate bowel preparation, with no differences in safety and tolerability compared with conventional preparation.

\section{Trial registration: ClinicalTrials.gov (NCT02300779).}

24 hours prior to the procedure. Patients with diabetes who are receiving hypoglycemic drug treatment should ensure sufficient carbohydrate intake in order to avoid hypoglycemia, and the dose of hypoglycemic drugs should be adjusted if the carbohydrate intake is reduced. However, these considerations are not taken into account in conventional bowel preparation protocols. Current clinical guidelines do not include specific recommendations for bowel preparation in patients with diabetes.

We hypothesized that a multifactorial strategy for bowel preparation in patients with diabetes, including an educational intervention with special attention to dietary recommendations and dose adjustment of blood glucose-lowering agents, could improve bowel cleansing and patient tolerability. 


\section{Patients and methods}

$\nabla$

The study protocol conformed to the ethical guidelines of the Declaration of Helsinki (2013 update), was approved by the Institutional review board of the Hospital del Mar, Barcelona (4511/I), and was registered at ClinicalTrials.gov (NCT02300779).

\section{Study design}

The study was a prospective, randomized, single-blind, parallel group, controlled trial to compare the efficacy, safety, and tolerability of a diabetes-specific preparation protocol with a conventional preparation for colon cleansing in patients with type 2 diabetes mellitus. A statistician generated the allocation sequence using a computer-generated block randomization table with a $1: 1$ allocation rate. Consecutive eligible patients were contacted by telephone for informed consent and invited to participate.

A research fellow randomized consenting patients to one of two bowel preparations, according to the randomization table, and assigned the bowel preparation protocol. At the time of the appointed colonoscopy, an investigator who was blinded to the bowel preparation protocol interviewed the patients. Five experienced endoscopists (>10000 colonoscopies each), who were blinded to the randomization, performed the colonoscopies with the patient under conscious sedation. Prior to the study, the endoscopists underwent a calibration exercise to improve consensus in the use of the Boston Bowel Preparation Scale (BBPS).

\section{Study population}

The study was conducted at the Hospital del Mar, Barcelona, between December 2014 and July 2015. Inclusion criteria were: consecutive patients with a previous diagnosis of type 2 diabetes mellitus under treatment with blood glucose-lowering agents, aged over 18 years, with scheduled outpatient screening, surveillance or diagnostic colonoscopy. Exclusion criteria were: unwillingness to participate, inability to follow instructions, previous colon resection surgery, incomplete colonoscopies for technical reasons, active intestinal bowel disease, and hospital admission.

\section{Interventions}

Patients were randomized to receive either a conventional bowel preparation (CBP, control group) or a diabetes-specific preparation protocol (DSP, intervention group). Patients assigned to the DSP group received a face-to-face visit at the hospital from a qualified nurse, who provided patient education with written and oral information regarding the importance of adequate bowel preparation, instructions on diet, laxative intake, and adjustment of blood glucose-lowering agents (see Appendix 1). A specific dietary plan designed by an endocrinologist and a registered dietitian consisted of a 4-day menu that specified low-fiber carbohydrate intake in each meal. A liquid diet was limited to 8 hours before the procedure; therefore, patients with a colonoscopy scheduled for the afternoon were allowed to have breakfast 3 hours before laxative intake on the same day as the colonoscopy.

The CBP for colonoscopy did not include an educational intervention. Patients were given written qualitative recommendations on a low-fiber diet for 3 days, starting 4 days before the procedure, and a clear liquid diet for 24 hours prior to colonoscopy. No specific recommendations on adjustments for blood glucoselowering agents were provided.

Both study groups received the same laxative protocol, which included $4 \mathrm{~L}$ of a polyethylene glycol solution (PEG) consumed as a split-dose regimen: $2 \mathrm{~L}$ in the evening of the preceding day and 2 $\mathrm{L}$ in the morning of the day of colonoscopy, starting 5 hours before the procedure.

\section{Outcome measures}

The primary outcome of the study was inadequate bowel preparation according to the BBPS. Colonoscopies with a score of less than 2 points in any segment were considered to be inadequate. Secondary outcomes included other indicators of colonoscopy quality: cecal intubation rate, overall and right-sided adenoma detection rate (ADR), as defined by the proportion of patients with at least one adenoma overall or in the right-side colon (proximal to the splenic flexure), respectively. These rates were computed using all patients undergoing a colonoscopy. Tolerability and volume perception were measured by means of a $1-10$ visual analog scale (VAS; 1 = excellent and $10=$ unbearable). Sideeffects evaluated were self-reported vomiting and bloating. Occurrence of hypoglycemic events was self-reported based on typical symptoms that resolved after the ingestion or administration of carbohydrates. Acceptability was defined as the willingness to repeat the same bowel preparation protocol in the future if needed.

\section{Data collection}

The following information was collected during a personal interview and from medical charts: age, sex, Eastern Cooperative Group (ECOG) performance status [6], Charlson Co-morbidity Index (CCI) [7], constipation, history of abdominal or pelvic surgery, previous colonoscopy, diabetes drug treatment, diabetes duration, late complications of diabetes (retinopathy, nephropathy, or neuropathy), and metabolic control measured by means of glycosylated hemoglobin $\left(\mathrm{HbA}_{1 \mathrm{c}}\right)$. Immediately before colonoscopy, patients completed, with the help of a research fellow, a questionnaire on tolerability, volume perception, referred sideeffects, and self-reported hypoglycemic episodes.

The timing of colonoscopy and the name of the endoscopist who performed the colonoscopy were recorded for each participant.

\section{Statistical analysis}

In a comparison of two proportions, a sample size of 79 patients was calculated to detect as statistically significant a minimum difference of $15 \%$ in the proportion of inadequate bowel preparation, from $20 \%$ in the CBP group to $5 \%$ in the DSP group, in a bilateral test, assuming an $\alpha$ risk of $5 \%$, statistical power of $80 \%$, and a maximum loss to follow-up of $5 \%$. The expected frequencies were obtained from the literature $[2,4,8]$ and our own unpublished previous data.

All patients who completed the preparation and underwent colonoscopy were included in the analysis except for those excluded for technical difficulties (as specified in the exclusion criteria). The outcome was inadequate bowel preparation and the predictors were bowel preparation protocol, sex, age, ECOG performance status, CCI, constipation, pelvic or abdominal surgery, previous colonoscopy, timing of colonoscopy, insulin treatment, diabetes mellitus duration, $\mathrm{HbA}_{1 \mathrm{c}}$, and late diabetes mellitus complications.

Qualitative variables were compared between groups by Pearson's chi-squared test. Fisher's exact test was used when one or more cells had an expected frequency of less than five. Quantitative variables were compared using the Student's $t$ test for independent samples. 


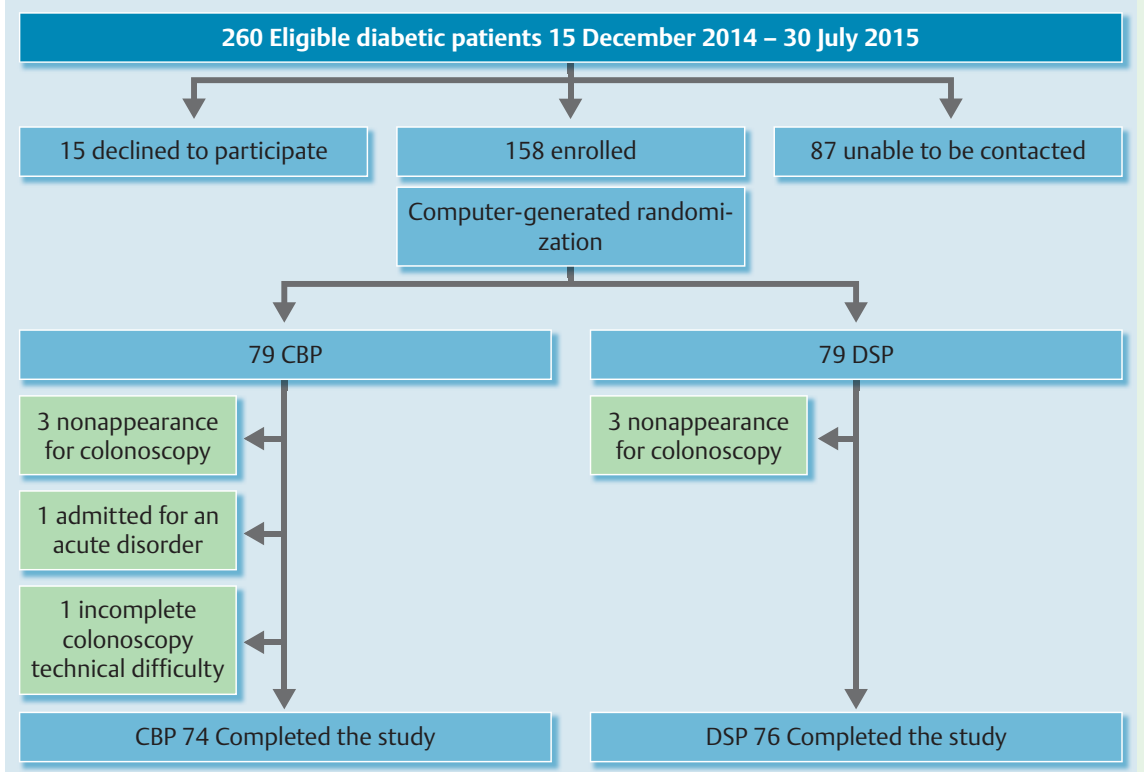

Fig. 1 Consort flow diagram of recruitment for patients with diabetes. CBP, conventional bowel preparation protocol; DSP, diabetes-specific preparation protocol.

A multivariate logistic regression model was applied to measure the effect of the intervention on the quality of bowel preparation, adjusting for potential confounders. All variables associated with the quality of preparation in the univariate analysis $(P<0.1)$ were included in the regression model. Two-tailed $P$ values of $<0.05$ were considered to be statistically significant. A research statistician performed the analysis using IBM SPSS software version 21 (IBM Corp., Armonk, New York, USA).

\section{Results}

The Consort flow diagram of patient recruitment is shown in - Fig.1. Of the 158 randomized patients, 8 (5\%) were excluded after randomization: 5 in the CBP group (3 for nonappearance, 1 incomplete colonoscopy due to a technical difficulty caused by bowel fixation in the sigmoid colon, and 1 patient was admitted for an ischemic stroke before starting the bowel preparation protocol), and 3 in the DSP group (all for nonappearance). Finally, 74 patients in the CBP group and 76 in the DSP group were included in the outcome analysis.

Both groups of patients were similar at entry and no differences were observed in colonoscopy indications ( Table 1 ). The main diagnoses following colonoscopy were adenomas (33\%), diverticulosis (21\%), and colorectal cancer (6\%).

In the DSP group, the mean time required for the educational intervention was 15 minutes (95\% confidence interval $[\mathrm{CI}] 13.9-$ 17.8).

\begin{tabular}{|c|c|c|c|}
\hline & $\begin{array}{l}\text { CPB } \\
(n=74)\end{array}$ & $\begin{array}{l}\text { DSP } \\
(n=76)\end{array}$ & $P$ \\
\hline Age, mean $(95 \% \mathrm{Cl})$, years & $70.6(68.3-72.9)$ & $69.1(67-71.3)$ & 0.35 \\
\hline Male sex, $n(\%)$ & $53(72)$ & $51(67)$ & 0.55 \\
\hline ECOG performance status > $1, n(\%)$ & $23(31)$ & $19(25)$ & 0.41 \\
\hline $\mathrm{CCl}>1, \mathrm{n}(\%)$ & $35(47)$ & $40(53)$ & 0.51 \\
\hline Constipation, n (\%) & $19(26)$ & $24(32)$ & 0.42 \\
\hline Pelvic/abdominal surgery, $\mathrm{n}(\%)$ & $24(32)$ & $19(25)$ & 0.31 \\
\hline Previous colonoscopy, n (\%) & $32(43)$ & $36(47)$ & 0.61 \\
\hline Timing of colonoscopy (afternoon), $n$ (\%) & $59(80)$ & $54(71)$ & 0.16 \\
\hline Insulin therapy, $\mathrm{n}(\%)$ & $17(23)$ & $27(36)$ & 0.09 \\
\hline Duration of diabetes, mean $(95 \% \mathrm{Cl})$ & $10.8(8.9-12.7)$ & $11.9(9.9-13.9)$ & 0.42 \\
\hline Late diabetes complications, $\mathrm{n}(\%)$ & $30(41)$ & $34(45)$ & 0.6 \\
\hline $\mathrm{HbA}_{1 \mathrm{c}}$, mean $(95 \% \mathrm{Cl}), \%$ & $7.2(6.9-7.5)$ & $7.2(6.9-7.5)$ & 0.75 \\
\hline \multicolumn{4}{|l|}{ Indications, n (\%) } \\
\hline Follow-up adenomas & $21(28)$ & $24(32)$ & 0.67 \\
\hline Anemia & $21(28)$ & $21(28)$ & 0.92 \\
\hline Hematochezia & $6(8)$ & $2(3)$ & 0.14 \\
\hline CRC screening & $6(8)$ & $9(12)$ & 0.45 \\
\hline Diarrhea & $4(5)$ & $7(9)$ & 0.37 \\
\hline Constipation & $4(5)$ & $7(9)$ & 0.37 \\
\hline
\end{tabular}

Table 1 Patient characteristics and indications for colonoscopy.

CBP, conventional bowel preparation protocol; SDP, diabetes-specific preparation protocol; $\mathrm{Cl}$, confidence interval; ECOG, Eastern Cooperative Group; $\mathrm{CCl}$, Charlson Co-morbidity Index; $\mathrm{HbA}_{1 c}$, glycosylated hemoglobin; $\mathrm{CRC}$, colorectal cancer. 


\begin{tabular}{|c|c|c|c|}
\hline & $\begin{array}{l}\text { CBP } \\
(n=74)\end{array}$ & $\begin{array}{l}\text { DSP } \\
(n=76)\end{array}$ & $P$ \\
\hline Inadequate bowel preparation, $\mathrm{n}(\%)$ & $15(20)$ & $5(7)$ & 0.014 \\
\hline \multicolumn{4}{|l|}{$\mathrm{BBPS}<2, \mathrm{n}(\%)$} \\
\hline Right colon & $14(19)$ & $5(7)$ & 0.023 \\
\hline Transverse colon & $11(15)$ & $3(4)$ & 0.022 \\
\hline Left colon & $11(15)$ & $3(4)$ & 0.022 \\
\hline Cecal intubation rate, $\mathrm{n}(\%)$ & $69(93)$ & $75(99)$ & 0.11 \\
\hline ADR, n (\%) & $24(32)$ & $25(33)$ & 0.95 \\
\hline Right-sided ADR, n (\%) & $15(20)$ & $21(28)$ & 0.29 \\
\hline High-risk adenoma, n (\%) & $12(16)$ & $13(17)$ & 0.88 \\
\hline Colorectal cancer, n (\%) & $6(8)$ & $3(4)$ & 0.28 \\
\hline Tolerability, VAS, mean $(95 \% \mathrm{Cl})$ & $5(4.3-5.7)$ & $4.1(3.3-4.8)$ & 0.075 \\
\hline Excessive volume perception, VAS, mean ( $95 \% \mathrm{Cl})$ & $5.9(5.2-6.5)$ & $5.4(4.7-6.1)$ & 0.38 \\
\hline Vomiting, n (\%) & $4(5)$ & $3(4)$ & 0.67 \\
\hline Bloating, n (\%) & $7(9)$ & $4(5)$ & 0.32 \\
\hline Symptomatic hypoglycemia, n (\%) & $3(4)$ & $1(1)$ & 0.3 \\
\hline Acceptability, n (\%) & $58(78)$ & $65(86)$ & 0.25 \\
\hline
\end{tabular}

Table 2 Comparison of primary and secondary outcomes between study groups.

CBP, conventional bowel preparation protocol; SDP, diabetes-specific preparation protocol; BBPS, Boston Bowel Preparation Scale; $A D R$, adenoma detection rate; VAS, visual analog scale ( 1 = excellent, $10=$ unbearable); $\mathrm{Cl}$, confidence interval.

\section{Primary outcome}

Bowel preparation was inadequate in more patients from the CBP group than from the DSP group ( $20 \%$ vs. $7 \%, P=0.014$; risk ratio $3.1,95 \% \mathrm{CI} 1.2-8)$. When the different colon segments were analyzed individually, similar differences between the study groups were obtained $(\bullet$ Table 2$)$.

\section{Secondary outcomes}

There were no differences in cecal intubation rate or in overall or right-sided ADR between the groups ( $\bullet$ Table 2 ). A nonsignificant trend towards better tolerability was observed in the DSP group $(P=0.075)$. Perception of volume, acceptability, and side-effects were similar between the study groups. Only four episodes of symptomatic hypoglycemia were reported: three in the CBP group and one in the DSP group $(P=0.3)$.

On univariate analyses, bowel preparation protocol, ECOG performance status, CCI, constipation, and late diabetes complications were associated with inadequate bowel preparation ( $\bullet$ Table3). On multivariate analysis, independent predictors of inadequate bowel preparation in patients with diabetes were CBP group (odds ratio [OR] 3.5, 95\%CI 1.2-10.4) and ECOG performance status $>1$ (OR 3.8, 95\%CI 1.4-10.2).

\section{Discussion}

In this randomized clinical trial, a multifactorial strategy for bowel preparation in patients with diabetes, which combined an educational intervention on dietary advice and adjustment of blood glucose-lowering agents, reduced the rate of inadequate bowel preparation threefold, with no statistically significant differences in safety and tolerability. In this respect, we wish to emphasize that this is the most ambitious study to date describing a successful strategy for improving bowel preparation in patients with diabetes mellitus.

Previous studies have reported that patients with diabetes had poorer bowel preparation, regardless of the laxative used, with an estimated prevalence of inadequate bowel preparation of $9 \%-30 \%[2-4,8]$. Thus, the prevalence of inadequate colon preparation observed in the CBP group of the present study concurs with these published data. These numbers are worrying because
1 in 5 colonoscopies in patients with diabetes would require a repeat procedure, with the associated diagnostic delay, risks for the patient, and costs for institutions. Few strategies to improve colon preparation in patients with diabetes have been published. An experimental trial compared the efficacy of two different laxative regimens for colon preparation in patients with diabetes. Although the study showed a statistically significant improvement (from 54\% good colon preparation in the standard group to $70 \%$ in the intervention group), the rate of inadequate bowel preparations in both groups was still high [9]. In another study in patients with diabetes, which evaluated the addition of lubiprostone to a single 4-L PEG dose, an improvement from $24 \%$ good or excellent bowel preparation to $47 \%$ was observed ( $P=$ 0.14 ), although it was statistically nonsignificant owing to the small sample size [10]. In both studies, the bowel preparation was started and finished on the day before the colonoscopy and given as a single-dose; current guidelines do not support any of these recommendations [11].

Current guidelines for colonoscopy preparation do not provide specific recommendations regarding dietary advice or modification of blood glucose-lowering agents for patients with diabetes. The rationale for our proposed multifactorial strategy for bowel preparation was based on three premises: a sensible dietary plan for a patient with diabetes, adjustment of blood glucoselowering agents to avoid hypoglycemia, and patient education to guarantee comprehension and reinforce adherence to recommendations.

Dietary recommendations for a patient with diabetes should ensure appropriate carbohydrate intake in order to avoid hypoglycemia. Therefore, our low-fiber dietary approach did not include a clear liquid diet on the day before the procedure, and patients were given strict qualitative and quantitative recommendations on carbohydrate intake.

The main concern with a 24-hour clear liquid diet in diabetes is the difficulty in ensuring appropriate carbohydrate intake and, consequently, maintaining glycemic control. Furthermore, some patients have altered gastrointestinal motility, which might contribute to delayed transit and constipation. The precise pathogenesis of these motility disturbances is unknown, although it has been suggested that they may be due to autonomic neuropathy of the gastrointestinal tract [12] or to hyperglycemia [13]. 


\begin{tabular}{|c|c|c|c|}
\hline & Adequate & Inadequate & $P$ \\
\hline$n(\%)$ & $20(13)$ & $130(87)$ & \\
\hline Bowel preparation, $\mathrm{n}(\%)$ & & & 0.014 \\
\hline $\mathrm{CBP}$ & $59(80)$ & $15(20)$ & \\
\hline DSP & $71(93)$ & $5(7)$ & \\
\hline Sex, n (\%) & & & 0.95 \\
\hline Male & $90(87)$ & $14(13)$ & \\
\hline Female & $40(87)$ & $6(13)$ & \\
\hline Age, mean $(95 \% \mathrm{Cl})$, years & $69.3(67.5-71.0)$ & $72.5(68.6-76.3)$ & 0.17 \\
\hline ECOG performance status, n (\%) & & & 0.004 \\
\hline $0-1$ & $99(92)$ & $9(8)$ & \\
\hline$>1$ & $31(74)$ & $11(26)$ & \\
\hline $\mathrm{CCl}, \mathrm{n}(\%)$ & & & 0.016 \\
\hline $0-1$ & $70(93)$ & $5(7 \%)$ & \\
\hline$>1$ & $60(80)$ & $15(20)$ & \\
\hline Constipation, $\mathrm{n}(\%)$ & & & 0.03 \\
\hline No & $93(90)$ & $10(10)$ & \\
\hline Yes & $33(77)$ & $10(23)$ & \\
\hline Pelvic/abdominal surgery, $\mathrm{n}(\%)$ & & & 0.34 \\
\hline No & $89(85)$ & $16(15)$ & \\
\hline Yes & $39(91)$ & $4(9)$ & \\
\hline Previous colonoscopy, n (\%) & & & 0.97 \\
\hline No & $71(87)$ & $11(13)$ & \\
\hline Yes & $59(87)$ & $9(13)$ & \\
\hline Timing of colonoscopy, n (\%) & & & 0.51 \\
\hline Morning & $30(83)$ & $6(17)$ & \\
\hline Afternoon & $99(88)$ & $14(12)$ & \\
\hline Insulin treatment, $\mathrm{n}(\%)$ & & & 0.94 \\
\hline No & $92(87)$ & $14(13)$ & \\
\hline Yes & $38(86)$ & $6(14)$ & \\
\hline Duration of diabetes, mean $(95 \% \mathrm{Cl})$, years & $11.2(9.7-12.7)$ & $12.8(8.9-16.7)$ & 0.43 \\
\hline $\mathrm{HbA}_{1 \mathrm{c}}$, mean $(95 \% \mathrm{Cl}), \%$ & $7.2(6.9-7.4)$ & $7.7(6.8-8.6)$ & 0.1 \\
\hline Late diabetes complications, $\mathrm{n}(\%)$ & & & 0.002 \\
\hline No & $81(94)$ & $5(6)$ & \\
\hline Yes & $49(77)$ & $15(23)$ & \\
\hline Endoscopist performing the procedure, $\mathrm{n}(\%)$ & & & 0.89 \\
\hline 1 & $31(89)$ & $4(11)$ & \\
\hline 2 & $39(89)$ & $5(11)$ & \\
\hline 3 & $10(83)$ & $2(17)$ & \\
\hline 4 & $27(82)$ & $6(18)$ & \\
\hline 5 & $23(89)$ & $3(12)$ & \\
\hline
\end{tabular}

Table 3 Univariate analysis of variables associated with inadequate bowel preparation in patients with diabetes mellitus.

CBP, conventional bowel preparation protocol; SDP, diabetes-specific preparation protocol; ECOG, Eastern Cooperative Group: $\mathrm{CCl}$ Charlson Co-morbidity Index; $\mathrm{HbA}_{1,}$, glycosylated hemoglobin.

In this respect, a clear liquid diet in patients with diabetes may further impair colon peristalsis and emptying of fecal matter. In addition, evidence of the efficacy of a clear liquid diet is limited and mostly derived from old studies of colon cleansing for radiography. Only one observational study in hospitalized patients has reported improved bowel preparation with a clear liquid diet [14]. However, a growing body of evidence has shown that a clear liquid diet is not beneficial [15-18]. Despite this, the latest guidelines of the US Multi-Society Task Force on Colorectal Cancer still recommend a full liquid diet in patients at high risk of inadequate colon preparation, such as those with diabetes mellitus [11]. One of the factors that could influence the quality of colon cleansing in the present study was the duration of the liquid diet: 24 hours in the CBP group and only 8 hours in the DSP group. A previous study showed that patients who were on a clear liquid diet the day before colonoscopy had less adherence to laxative intake and worse quality of bowel cleansing [18].

To reduce the risk of hypoglycemiain in the present study, we included recommendations on dose adjustments for blood glucose- lowering agents. However, as the dietary intervention did not reduce carbohydrate intake, modifications of the diabetes therapy were restricted to the day of the procedure. Although hypoglycemic therapy adjustment is commonly recommended for other ambulatory procedures that limit dietary intake, such as ambulatory surgery [19], current clinical guidelines for colonoscopy preparation do not include specific recommendations.

Most patients refer to bowel preparation as the most difficult and unpleasant part of colonoscopy [20], and adherence to bowel preparation instructions is critical for the quality of the procedure. In this regard, previous studies revealed [21 - 24] the benefits of an educational intervention for bowel cleansing, although none of them specifically included patients at high risk for inadequate colon preparation, such as those with diabetes. Given the fact that these patients are older, with more co-morbidity and often complex drug treatments, an educational intervention would be expected to be appropriate to improve bowel cleansing. Nevertheless, no clinical trials had previously confirmed this hypothesis. In the present study, the educational intervention re- 


\section{Competing interests: None}

\section{Acknowledgments}

The authors thank Miss Christine O'Hara for review of the English version of the manuscript and Mr. Sergio Mojal who provided statistical advice.

\section{References}

1 Sun $L, Y u$ S. Diabetes mellitus is an independent risk factor for colorectal cancer. Dig Dis Sci 2012; 57: 1586-1597

2 Taylor C, Schubert ML. Decreased efficacy of polyethylene glycol lavage solution (golytely) in the preparation of diabetic patients for outpatient colonoscopy: a prospective and blinded study. Am J Gastroenterol 2001; 96: 710-714

3 Ozturk NA, Gokturk HS, Demir M et al. The effect of autonomous neuropathy on bowel preparation in type 2 diabetes mellitus. Int J Colorectal Dis 2009; 24: 1407-1412

4 Dik VK, Moons LMG, Hüyük M et al. Predicting inadequate bowel preparation for colonoscopy in participants receiving split-dose bowel preparation: Development and validation of a prediction score. Gastrointest Endosc 2015; 81: 665-672

5 Froehlich F, Wietlisbach V, Gonvers J-J et al. Impact of colonic cleansing on quality and diagnostic yield of colonoscopy: the European Panel of Appropriateness of Gastrointestinal Endoscopy European multicenter study. Gastrointest Endosc 2005; 61: 378-384

6 Oken MM, Creech RH, Tormey DC et al. Toxicity and response criteria of the Eastern Cooperative Oncology Group. Am J Clin Oncol 1982; 5: 649-655

7 Charlson ME, Pompei P, Ales KL et al. A new method of classifying prognostic comorbidity in longitudinal studies: development and validation. J Chronic Dis 1987; 40: 373-383

8 Ozturk NA, Gokturk HS, Demir M et al. Efficacy and safety of sodium phosphate for colon cleansing in type 2 diabetes mellitus. South Med J 2010; 103: 1097 - 1102

9 Hayes A, Buffum $M$, Hughes J. Diabetic colon preparation comparison study. Gastroenterol Nurs 2011; 34: 377-382

10 Grigg E, Schubert MC, Hall J et al. Lubiprostone used with polyethylene glycol in diabetic patients enhances colonoscopy preparation quality. World J Gastrointest Endosc 2010; 2: 263-267

11 Johnson DA, Barkun AN, Cohen LB et al. Optimizing adequacy of bowel cleansing for colonoscopy: recommendations from the US Multi-Society Task Force on Colorectal Cancer. Am J Gastroenterol 2014; 109: $1528-1545$

12 Battle WM, Snape WJ, Alavi A et al. Colonic dysfunction in diabetes mellitus. Gastroenterology 1980; 79: 1217-1221
13 Sims MA, Hasler WL, Chey WD et al. Hyperglycemia inhibits mechanoreceptor-mediated gastrocolonic responses and colonic peristaltic reflexes in healthy humans. Gastroenterology 1995; 108: 350-359

14 Reilly $T$, Walker $G$. Reasons for poor colonic preparation with inpatients. Gastroenterol Nurs 2004; 27: 115-117

15 Jung YS, Seok HS, Park DI et al. A clear liquid diet is not mandatory for polyethylene glycol-based bowel preparation for afternoon colonoscopy in healthy outpatients. Gut Liver 2013; 7: 681-687

16 Park DI, Park SH, Lee SK et al. Efficacy of prepackaged, low residual test meals with $4 \mathrm{~L}$ polyethylene glycol versus a clear liquid diet with $4 \mathrm{~L}$ polyethylene glycol bowel preparation: a randomized trial. J Gastroenterol Hepatol 2009; 24: 988 - 991

17 Sipe BW, Fischer $M$, Baluyut AR et al. A low-residue diet improved patient satisfaction with split-dose oral sulfate solution without impairing colonic preparation. Gastrointest Endosc 2013; 77: 932 - 936

18 Soweid AM, Kobeissy AA, Jamali FR et al. A randomized single-blind tria of standard diet versus fiber-free diet with polyethylene glycol electrolyte solution for colonoscopy preparation. Endoscopy 2010; 42: $633-638$

19 Joshi GP, Chung F, Vann MA et al. Society for Ambulatory Anesthesia consensus statement on perioperative blood glucose management in diabetic patients undergoing ambulatory surgery. Anesth Analg 2010; 111: $1378-1387$

$20 \mathrm{Ko} C W$, Riffle S, Shapiro JA et al. Incidence of minor complications and time lost from normal activities after screening or surveillance colonoscopy. Gastrointest Endosc 2007; 65: 648 - 656

21 Abuksis G, Mor M, Segal N et al. A patient education program is cost-effective for preventing failure of endoscopic procedures in a gastroenterology department. Am J Gastroenterol 2001; 96: 1786 - 1790

22 Tae JW, Lee JC, Hong SJ et al. Impact of patient education with cartoon visual aids on the quality of bowel preparation for colonoscopy. Gastrointest Endosc 2012; 76: 804-811

23 Spiegel BMR, Talley J, Shekelle P et al. Development and validation of a novel patient educational booklet to enhance colonoscopy preparation. Am J Gastroenterol 2011; 106: 875-883

24 Liu X, Luo H, Zhang L et al. Telephone-based re-education on the day before colonoscopy improves the quality of bowel preparation and the polyp detection rate: a prospective, colonoscopist-blinded, randomised, controlled study. Gut 2014; 63: 125 - 130

25 Harewood GC, Sharma VK, de Garmo P. Impact of colonoscopy preparation quality on detection of suspected colonic neoplasia. Gastrointest Endosc 2003; 58: 76-79

26 Jover R, Zapater P, Polanía E et al. Modifiable endoscopic factors that influence the adenoma detection rate in colorectal cancer screening colonoscopies. Gastrointest Endosc 2013; 77: 381 - 389.e1

27 Calderwood $A H$, Jacobson BC. Comprehensive validation of the Boston Bowel Preparation Scale. Gastrointest Endosc 2010; 72: 686-692 